\title{
Analysis of the dependence of the parameters of the generalized Peukert's equations on the capacity for nickel-cadmium batteries
}

\author{
Nikolay E. Galushkin ${ }^{1,}$, Dmitriy N. Galushkin ${ }^{1}$, Nataliya N. Yazvinskaya ${ }^{1}$, and \\ Sergei P. Gukov ${ }^{1}$ \\ ${ }^{1}$ Don State Technical University, Laboratory of electrochemical and hydrogen energy, 346500 \\ Shakhty, Russia
}

\begin{abstract}
In this paper, the use possibility was analyzed of the most wellknown generalized Peukert's equations, for computing of released capacity of nickel-cadmium batteries at different discharge currents. It was proved that these equations correspond well to experimental data throughout the entire variation interval of discharge currents. It was shown that the parameter $\mathrm{n}$ does not depend on a nominal capacity of a batteries under examination. Farther, it was shown that a functional dependence of a battery's released capacity with a discharge current is determined by the statistical phase transition subjected to the normal distribution law.
\end{abstract}

\section{Introduction}

Virtually all the contemporary technical devices contain batteries in their structure. Hence for these devices design engineering and their work optimization, highly reliable models of batteries are needed.

The Peukert's equation was one of the first analytic equations describing processes in batteries [1]

$$
C=\frac{A}{i^{n}}
$$

where $\mathrm{C}$ is capacity released by a battery at the discharge current $\mathrm{I}$, while A and $\mathrm{n}$ are empiric constants. However even now, the Peukert's equation is widely used in various models. For example, in the papers [2,3], the Peukert's equation was used as a component part of statistical models for evaluation of a remaining capacity in lithium-ion batteries.

Besides often, the Peukert's equation is used as one of possible criteria for a validation of fundamental electrochemical models of batteries [4-8] and non-linear structural models [911]. It should be observed that electrochemical models of batteries are not always acceptable for a practical use [2]. A use of this kind of models requires a knowledge of a lot number of local parameters, which - at any system changes - are necessary to be corrected. Besides, they are very sophisticated. Consequently for their solutions, it require high

\footnotetext{
* Corresponding author: galushkinne@mail.ru
} 
computation-intensive power, which is not acceptable for on-board computers of airplanes and electromobiles $[12,13]$. That is why very often at building of practical models of batteries, statistical models are used [2,3,12-15]. Also the statistical models are used, when there is a need in modeling of such poorly studied phenomena in batteries as the thermal runaway $[16,17]$ or the hydrogen accumulation in electrodes $[18,19]$.

This study is aimed at analysis of parameters variation of the generalized Peukert's equations for batteries of various modes of discharge as these equations are used very often in different models of batteries [2,3].

\section{Generalized Peukert's equations}

According to the Peukert's equation (1), a battery's released capacity tends to infinity with discharge current decrease. So the Peukert's equation is not applicable at small discharge currents. At present time, there exist a lot of Peukert's equations generalizations making away with this problem [20-22].

$$
\begin{gathered}
C=\frac{A}{1+B i^{n}}, \\
C=\frac{A}{i^{n}} \tanh \left(\frac{i^{n}}{B}\right) .
\end{gathered}
$$

A process of batteries discharge is a phase transition, whereas often phase transitions are described by the complementary error function $[22,23]$

$$
C=\frac{A}{2} \cdot \operatorname{erfc}\left(\frac{i-i_{k}}{\sqrt{2} \sigma}\right)
$$

where $\sigma$ is standard deviation and $i_{k}$ is mean value of the statistical variable $i$.

Of course also, there exist other equations and methods for battery's released capacity computing at different discharge currents [20, 24-26]. However in the papers [21,22], it was shown that the empiric equations (2-4) are most appropriate to the experimental data throughout the entire interval of discharge currents variation including at small discharge currents. That is why in this paper, we shall study only these equations.

For analysis convenience's sake, we shall rewrite the (2) in the following form

$$
C=\frac{C_{m}}{1+\left(\frac{i}{i_{0}}\right)^{n}} .
$$

Then $C_{m}$ is battery's top capacity (obtained at small discharge currents because $C(0)=C_{m}$ ), while $i_{0}$ is the current, at which the battery releases twice less capacity than its top capacity because $C\left(i_{0}\right)=C_{m} / 2$. Hence in the equation (5), the constants have a clear electrochemical sense unlike the equation (2), where $A, B$ are just empiric constants.

Let us rewrite also the equations $(3,4)$ in the following form

$$
C=\frac{0.522 C_{m}}{\left(\frac{i}{i_{0}}\right)^{n}} \tanh \left(\left(\frac{i}{i_{0}}\right)^{n} \frac{1}{0.522}\right),
$$




$$
C=\frac{C_{m}}{\operatorname{erfc}\left(\frac{-1}{n}\right)} \cdot \operatorname{erfc}\left(\frac{i / i_{k}-1}{n}\right)
$$

Into the equation (6), there were added the constants 0.522 in order that the parameters $C_{m}$ and $i_{0}$ had the same electrochemical sense as in the equation (5). For the equation (7), the conditions $C(0)=C_{m}$ and $C\left(i_{k}\right)=C_{m} / \operatorname{erfc}(-1 / n)$ will be fulfilled. Hence also for the equation (7), $C_{m}$ is a top capacity of a battery, whereas $i_{k}$ is the current value, at which the battery releases a capacity $\operatorname{erfc}(-1 / n)$ times less than its top capacity. In the sequel, it will be shown that - as a rule $-\mathrm{n} \leq 1$, in this case $1.85 \leq \operatorname{erfc}(-1 / n) \leq 2$.

It should be observed that the parameters $i_{k}$ and $\sigma$ in the equation (4) have the wellknown statistical sense. From comparison between the equations (4) and (7), it follows that $\sqrt{2} \sigma=i_{k} n$. Hence in the rewritten equations (5-7), all the constants have a clear electrochemical or statistical sense unlike the initial empiric equations (2-4).

\section{Experimental}

For the parameters evaluation of the equations (5-7), batteries SBLE made by SAFT the company (of stationary use) with pocket electrodes.

Batteries' discharging was performed down to the voltage $1 \mathrm{~V}$. In the experiments, there were used the discharge currents from $0.1 C_{N}$ (where $C_{N}$ is a battery's nominal capacity) up to currents, at which the battery discharge capacity was close to zero. Batteries' charging was performed according to batteries operation manuals.

In order to avoid a mutual influence of charge-discharge cycles (via various remaining phenomena, in particular, memory effect, etc.), before each discharge current change, training cycles were fulfilled. The training cycles were performed until in three cycles without interruption, the released capacity started differing less than by $5 \%$. The training cycles were performed according to operation manuals of the batteries under examination.

As a released capacity value at a specific discharge current, an average capacity value was taken in three charge-discharge cycles in succession. However if in these three cycles the released capacity differed more than by $5 \%$, additional training cycles were performed and the experiment was repeated again.

It should be observed that for batteries of the same nominal capacity, their released capacity depends on a lot of random factors such as statistical dispersion of batteries' parameters at their manufacturing, their operation life, their operation modes, etc. Our experience with batteries cycling showed that in a lot of batteries of the same kind, under the same discharge current, their released capacity can differ by 4 or $5 \%$ and more. This relates to batteries of any electrochemical systems and is due to the above mentioned random factors. That is why if to standardize the obtained experimental data for batteries' capacity by their top capacity - found in experiments for a specific battery, - in this case, the above mentioned random factors will be obviated to a large extent. This method allows finding empiric experimental curves more reliably.

\section{Results and discussion}

In the experiments, there were used the batteries of production by SAFT the company, of stationary use with pocket electrodes: SBLE 15, SBLE 95, SBLE 230 (with capacities 15, 95, 230Ah respectively. 
The obtained experimental data (in the standardized coordinates) for the batteries under examination are represented in the Figure 1. As the parameter $C_{m}$ on the Figure 1, the experimental values were taken of batteries' released capacities found at the discharge current $0.1 \mathrm{C}_{\mathrm{N}}$. As a result of such calibration, locations of experimental points on the Figure 1 are the same regardless of the studied equations. The parameters $i_{0}, i_{k}$ on the Figure 1 were taken from Table 1 for each of generalized Peukert's equations (5-7) respectively.

For the generalized Peukert's equations (5-7), optimal parameters were found with use of the least square method and Levenberg-Marquardt optimization procedure. The obtained optimal parameters are represented in the Table 1.

From the Figure 1, it is seen that for batteries of different capacities (in the standardized coordinates), the optimal experimental curves coincide in limits of the standard error. That is why on the Figure 1 for each of the equations (5-7), only one curve is represented corresponding to the average capacity of the batteries under investigation.

Based on the analysis of the Table 1, one can come to the following conclusions.

Firstly, all the studied generalized Peukert's equations (5-7) can be used for the evaluation of a battery's released capacity as in the case of these equations use, the relative error of experimental data approximation is less than $6 \%$. As a rule, this error is acceptable for a practical evaluation of remaining capacity in a battery [2]. However, it should be observed that the equations $(5,7)$ correspond to experimental data best of all as they have the least error of approximation.

Secondly, the parameter $n$ in the generalized Peukert's equations (5-7) does not depend on a batteries capacity as the found values coincide in limits of the standard error. (Table 1). This is due to the fact that in the standardized coordinates, the equations (5-7) depend only on one parameter, namely on $n$. Hence in the case of the coincidence of the experimental data (Fig. 1), also this parameter is expected to make the coincidence. The parameters $C_{m}$, $i_{0}, i_{k}$ depend on batteries' capacity (Table 1 ).

From a theoretical point of view, most interesting one is the equation (7). The equation (7) has a statistical fundament unlike the equations $(5,6)$, which are purely empiric equations.

The process of batteries discharge is a phase transition. For example, for positive electrodes during discharge, the phase transition goes from more oxidized phases of active matter to less oxidized phases, whereas for negative electrodes it is on contrary.

$$
\begin{gathered}
\mathrm{NiOOH}+\mathrm{H}_{2} \mathrm{O}+\mathrm{e}^{-} \rightarrow \mathrm{Ni}(\mathrm{OH})_{2}+\mathrm{OH}^{-} \\
\mathrm{Cd}+2 \mathrm{OH}^{-} \rightarrow \mathrm{Cd}(\mathrm{OH})_{2}+2 \mathrm{e}^{-} \quad \text { (anode) }
\end{gathered}
$$

In physics often $[22,23]$, the phase transitions are described by the complementary error function (4). The complementary error function (4) is based on the normal distribution law. It is beyond doubt that on the level of molecules and ions, the discharge process is a statistical process. Hence from a good coincidence of the experimental data with the equation (7) (Fig.1), it is possible to conclude that the process of batteries discharge is a statistical process subjected to the normal distribution law. 
Table 1. Optimal parameters of generalized Peukert's equations (5-7) for batteries with pocket electrodes and long discharge mode.

\begin{tabular}{|c|c|c|c|}
\hline \multirow{2}{*}{ Equation Parameters } & \multicolumn{3}{|c|}{ batteries } \\
\hline & SBLE 15 & SBLE 95 & SBLE 230 \\
\hline \multicolumn{4}{|c|}{ Equation (5) } \\
\hline$C_{m}(\mathrm{Ah})$ & 15.207 & 97.139 & 235.846 \\
\hline Standard error for $C_{m}(\mathrm{Ah})$ & 0.230 & 1.343 & 3.885 \\
\hline$i_{0}(\mathrm{~A})$ & 11.644 & 67.963 & 159.272 \\
\hline Standard error for $i_{0}$ (A) & 0.258 & 1.341 & 3.693 \\
\hline$n$ & 3.071 & 3.082 & 3.095 \\
\hline Standard error for $n$ & 0.159 & 0.137 & 0.160 \\
\hline Mean $n$ & 3.083 & 3.083 & 3.083 \\
\hline $\mathrm{SD}^{\mathrm{a}}$ & 0.266 & 1.562 & 4.17 \\
\hline$\delta^{b}(\%)$ & 3.691 & 3.472 & 3.861 \\
\hline \multicolumn{4}{|c|}{ Equation (6) } \\
\hline$C_{m}$ (Ah) & 14.984 & 95.708 & 232.568 \\
\hline Standard error for $C_{m}(\mathrm{Ah})$ & 0.344 & 2.046 & 5.279 \\
\hline$i_{0} \quad(\mathrm{~A})$ & 11.434 & 66.521 & 155.515 \\
\hline Standard error for $i_{0}(\mathrm{~A})$ & 0.393 & 2.043 & 4.964 \\
\hline$n$ & 1.988 & 2.019 & 2.032 \\
\hline Standard error for $n$ & 0.161 & 0.144 & 0.150 \\
\hline Mean $n$ & 2.013 & 2.013 & 2.013 \\
\hline SD & 0.420 & 2.398 & 5.951 \\
\hline$\delta$ & 5.833 & 5.33 & 5.950 \\
\hline \multicolumn{4}{|c|}{ Equation (7) } \\
\hline$C_{m}(\mathrm{Ah})$ & 16.212 & 104.313 & 253.400 \\
\hline Standard error for $C_{m}(\mathrm{Ah})$ & 0.204 & 1.793 & 5.219 \\
\hline$i_{k}(\mathrm{~A})$ & 10.862 & 62.057 & 145.430 \\
\hline Standard error for $i_{k}$ (A) & 0.370 & 3.174 & 8.906 \\
\hline$n$ & 1.032 & 1.074 & 1.081 \\
\hline Standard error for $n$ & 0.077 & 0.115 & 0.136 \\
\hline Mean $n$ & 1.062 & 1.062 & 1.062 \\
\hline SD & 0.206 & 1.307 & 3.287 \\
\hline$\delta$ & 2.859 & 2.914 & 3.044 \\
\hline
\end{tabular}




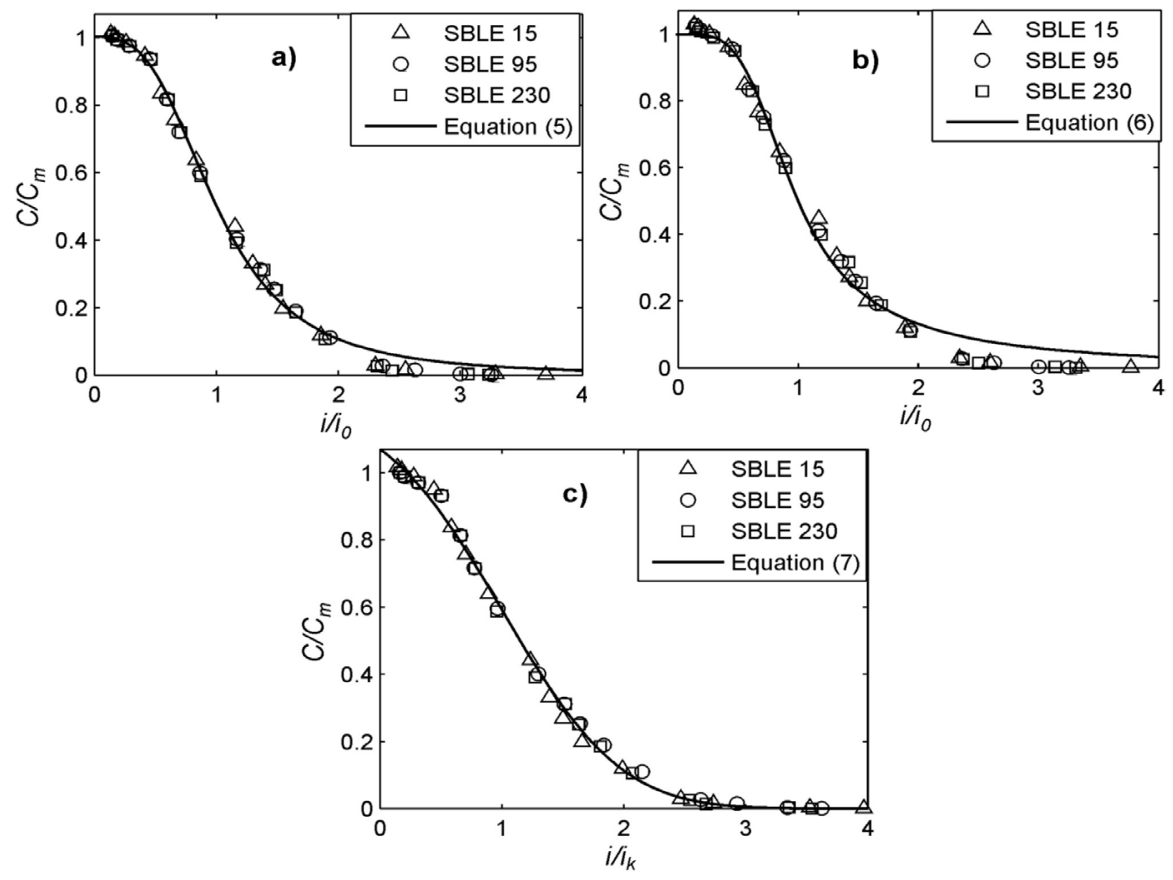

Fig. 2. Experimental data for the batteries of long discharge mode with pocket electrodes and the generalized Peukert's equations (5-7) (a,b,c respectively). $\mathrm{Cm}$ is top capacity of batteries found at discharge current $0.1 \mathrm{C}_{\mathrm{N}} . i_{0}$ is the current, at which batteries release capacity twice less than their top capacity. $i_{k}$ is the current, at which batteries release capacity $\operatorname{erfc}(-1 / n)$ times less than their top capacity. The values of the parameters $i_{0}, i_{k}$ were taken from the Table 1 for each of the generalized Peukert's equations (5-7) respectively.

In our opinion, this experimental fact is very significant for a theoretical substantiation of charge/discharge processes in batteries.

So the proposed statistical mechanism of the process of batteries discharge has a clear both physical and electrochemical sense and allows explaining the variation of the parameters in the equations (5-7) depending on a type of batteries in use.

\section{Conclusion}

In conclusion, I would list a number of advantages of the proposed generalized Peukert's equation (7) as compared to both the classical Peukert's equation (1) and the equations $(5,6)$.

Firstly, the generalized Peukert's equation (7) has the clear both statistical and electrochemical sense unlike the equations $(1,5,6)$, which are purely empiric equations. This statistical mechanism of the process of batteries discharge allows explaining the parameters variation of the equation (7) depending on a type of used batteries.

Secondly, both the equation (7) and the equation (5) have the least error of approximation of experimental data (less than $4 \%$ ), which is quite enough for a practical evaluation of a battery's released capacity.

As different generalizations of the Peukert's equation are widely used in various evaluations and models [2,3], the correction of those equations and discovering of the physical and electrochemical mechanism, which they are based on, have a great both practical and theoretical significance. 


\section{References}

1. H. Wenzl, J. Garche, Encyclopedia of Electrochemical Power Sources (Elsevier, Amsterdam, 2009)

2. A. Hausmann and C. Depcik, J. Power Sources, 235, 148 (2013)

3. N.E. Galushkin, N.N. Yazvinskaya, D.N. Galushkin, J. Electrochem. Soc., 162, A308 (2015)

4. M. Cugnet, S. Laruelle, S. Grugeon, B. Sahut, J. Sabatier, J.M. Tarascon, A. Oustaloup, J. Electrochem. Soc., 156, A974 (2009)

5. K. Siniard, M. Xiao and S. Y. Choe, J. Power Sources, 195, 7102 (2010)

6. M. Venkatraman and J. W. Van Zee, J. Power Sources, 166, 537 (2007)

7. T.G. Zavalis, M. Behm, G. Lindbergh, J. Electrochem. Soc., 159, A848 (2012)

8. V. Boovaragavan, R.N. Methakar, V. Ramadesiga, V.R. Subramanian, J. Electrochem. Soc., 156, A854 (2009)

9. N.E. Galushkin, N.N. Yazvinskaya, D.N. Galushkin, Int. J. Electrochem. Sci., 9, 6305 (2014)

10. N.E. Galushkin, N.N. Yazvinskaya, D.N. Galushkin, J. Electrochem. Soc., 159, A1315 (2012)

11. N.E. Galushkin, N.N. Yazvinskaya, D.N. Galushkin, ECS Electrochem. Lett., 4, A94 (2015)

12. O. Tremblay, L.A. Dessaint, A.I. Dekkiche, Vehicle Power and Propulsion Conference, VPPC 2007 (IEEE, Arlington, 2007)

13. O. Tremblay, L.A. Dessaint, World Electric Vehicle Journal, 3, 1 (2009)

14. 14. N.E. Galushkin, N.N. Yazvinskaya, D.N. Galushkin, I.A. Galushkina, Int. J. Electrochem. Sci., 10, 5530 (2015)

15. N.E. Galushkin, N.N. Yazvinskaya, D.N. Galushkin, Int. J. Electrochem. Sci., 13, 1275 (2018)

16. N.E. Galushkin, N.N. Yazvinskaya, D.N. Galushkin, Int. J. Electrochem. Sci., 162, A749 (2015)

17. N.E. Galushkin, N.N. Yazvinskaya, D.N. Galushkin, J. Electrochem. Soc., 162, A2044 (2015)

18. N.E. Galushkin, N.N. Yazvinskaya, D.N. Galushkin, I.A. Galushkina, Int. J. Electrochem. Sci., 10, 6645 (2015)

19. N.E. Galushkin, N.N. Yazvinskaya, D.N. Galushkin, Int. J. Hydrogen Energy, 41, 14813 (2016)

20. J. Garche, Encyclopedia of Electrochemical Power Sources (Elsevier, Amsterdam, 2009)

21. N.E. Galushkin, N.N. Yazvinskaya, D.N. Galushkin, Int. J. Electrochem. Sci., 9, 1911 (2014)

22. N.E. Galushkin, N.N. Yazvinskaya, D.N. Galushkin, I.A. Galushkina, Int. J. Electrochem. Sci., 9, 4429 (2014)

23. L.P. Pitaevskii, E.M. Lifshitz, Physical Kinetics, 10, 522, (1981)

24. L.H. Saw, K. Somasundaram, Y. Ye, A.O. Tay, J. Power Sources, 249, 231 (2014)

25. S.M. Rezvanizaniani, Z. Liu, Y. Chen, J. Lee, J. Power Sources, 256, 110 (2014) 